\title{
Correlations between personality traits and specific groups of alpha waves in the human EEG
}

\author{
Tomas Johannisson Corresp. 1 \\ ${ }^{1}$ Department of Psychiatry, Sahlgrenska University Hospital, Mölndal, Sweden \\ Corresponding Author: Tomas Johannisson \\ Email address: tomas.johannisson@outlook.com
}

Background. Different individuals have alpha waves with different wavelengths. The distribution of the wavelengths is assumed to be bell-shaped and smooth. Although this view is generally accepted, it is still just an assumption and has never been critically tested. When exploring the relationship between alpha waves and personality traits, it makes a huge difference if the distribution of the alpha waves is smooth or if specific groups of alpha waves can be demonstrated. Previous studies have not considered the possibility that specific groups of alpha waves may exist.

Methods. Computerized EEGs have become standard, but wavelength measurements are problematic when based on averaging procedures using the Fourier transformation because such procedures cause a large systematic error. If the actual wavelength is of interest, it is necessary to go back to basic physiology and use raw EEG signals. In the present study, measurements were made directly from sequences of alpha waves where every wave could be identified. Personality dimensions were measured using an inventory derived from the International Personality Item Pool.

Results. Recordings from 200 healthy individuals revealed that there are three main groups of alpha waves. These groups had frequencies around 8,10 , and 12 waves per second. The middle group had a bimodal distribution, and a subdivision gave a total of four alpha groups. In the center of each group, the degree of extraversion was high and the degree of neuroticism was low. Many small differences in personality traits were found when the centers were compared with one another. This gave four personality profiles that resemble the four classical temperaments. When people in the surrounding zones were compared with those in the centers, relatively large differences in personality traits were found.

Conclusions. Specific groups of alpha waves exist, and these groups have to be taken into account when correlations are made to personality dimensions and temperament types. There is a link between alpha waves and personality traits, and this link implies that there is an underlying relationship. To explain the nature of this relationship, there are two hypotheses that can be applied. One of these deals with the general organization of the forebrain and the other explains why the brain generates alpha waves. 


\section{Correlations between personality traits and specific groups

Tomas Johannisson

Email: tomas.johannisson@outlook.com 


\section{ABSTRACT}

8 Background. Different individuals have alpha waves with different wavelengths. The

9 distribution of the wavelengths is assumed to be bell-shaped and smooth. Although this view

10 is generally accepted, it is still just an assumption and has never been critically tested. When

11 exploring the relationship between alpha waves and personality traits, it makes a huge

12 difference if the distribution of the alpha waves is smooth or if specific groups of alpha waves

13 can be demonstrated. Previous studies have not considered the possibility that specific groups

14 of alpha waves may exist.

15 Methods. Computerized EEGs have become standard, but wavelength measurements are

16 problematic when based on averaging procedures using the Fourier transformation because

17 such procedures cause a large systematic error. If the actual wavelength is of interest, it is

18 necessary to go back to basic physiology and use raw EEG signals. In the present study,

19 measurements were made directly from sequences of alpha waves where every wave could be

20 identified. Personality dimensions were measured using an inventory derived from the

21 International Personality Item Pool.

22 Results. Recordings from 200 healthy individuals revealed that there are three main groups of

23 alpha waves. These groups had frequencies around 8, 10, and 12 waves per second. The middle

24 group had a bimodal distribution, and a subdivision gave a total of four alpha groups. In the center of each group, the degree of extraversion was high and the degree of neuroticism was low. Many small differences in personality traits were found when the centers were compared with one another. This gave four personality profiles that resemble the four classical temperaments. When people in the surrounding zones were compared with those in the centers, relatively large differences in personality traits were found.

30 Conclusions. Specific groups of alpha waves exist, and these groups have to be taken into account when correlations are made to personality dimensions and temperament types. There is a link between alpha waves and personality traits, and this link implies that there is an underlying relationship. To explain the nature of this relationship, there are two hypotheses that can be applied. One of these deals with the general organization of the forebrain and the other explains

35 why the brain generates alpha waves. 


\section{Alpha waves and personality dimensions}

Many researchers have attempted to define the relationship between alpha waves and personality dimensions (Gale, 1983; Klimesch, 1999). Of all the data obtained via electroencephalography (EEG), individual alpha frequency stands out as a measure that can be correlated with personality dimensions. For instance, Robinson (2001) found 11 studies that reported a link between alpha frequency and the degree of extraversion. In all of these studies, the mean value of the alpha frequency was slightly higher in groups with introverted individuals compared to groups with extraverted individuals. The differences were statistically significant in seven of the studies.

Over the years, controversies have emerged regarding the various factors that could influence the link between alpha waves and extraversion (Gale, 1983; Tran, Craig \& McIssac, 2001; Robinson, 2001). In a study conducted by Hagemann et al. (2009), internal and external factors were found to have a minimal effect on this correlation. The authors tested whether there was a linear relationship between alpha power density and extraversion, and they found that the correlation coefficient for the overall measure was small $(0.22)$. None of the previous studies tested for a relationship along curved lines or took into consideration that there might be specific alpha groups (Gale, 1983; Klimesch, 1999; Tran, Craig \& McIssac, 2001; Robinson, 2001;

54 Hagemann et al., 2009).

\section{Need for an alpha hypothesis}

To understand how alpha waves are linked to personality traits, it would be helpful to have an explanation as to why the brain generates alpha waves. A possible explanation involves the activity in the columns in the cerebral cortex. It has been suggested that each column exhibits neuronal activity either at a high level or at a comparatively low level (Johannisson, 1984).

In this model, a cerebral column has activity at the high level for a short period of time. When the activity in a column changes from the high level to the low level, the activity in another column may jump up to the high level. Thus, there is a continuous turnover of highly active columns, which results in a flow of thoughts and feelings.

There is a difference in mental activity when something is appearing in consciousness and when that something has disappeared from the conscious level. Two different levels of neuronal activity may account for this observation. Furthermore, only a very tiny fraction of everything that could appear in consciousness actually appears there at each moment in time. To account for this, it is likely that only a very small number of columns are involved in high-level processing at any one time (Johannisson, 1993). 
70 In the "two levels" hypothesis, the number of columns active at the high level is kept within 71 certain limits via a regulating system (Johannisson, 1984). When the number of highly active 72 columns is at the lower limit, regulating signals from the thalamus raise the excitability of all 73 columns that are active at the low level until one of them jumps up to the high level of activity.

74 Conversely, when the upper limit is reached, the regulating system decreases the excitability.

75 When one of the highly active columns leaves the high conscious level, the system returns to the

76 lower limit, such that the excitability will be increased again. These repeated changes in

77 excitability in all columns with low activity are seen in the EEG as alpha waves. This hypothesis

78 explains why and how alpha waves are generated (Johannisson \& Nilsson, 1996).

\section{Groups of alpha waves}

80 The limits for the number of highly active columns can be different for different individuals

81 (Johannisson \& Nilsson, 1996). Therefore, if the hypothesis is true, there must be specific

82 frequency groups corresponding to the different limits. However, alpha frequencies from

83 different individuals are usually described as having a distribution that is smooth and

84 homogeneous (Klimesch, 1999; Robinson, 2006; Başar, 2012; Bazanova \& Vernon, 2014). This

85 is an assumption that has become generally accepted, although it has never been critically tested.

86 In the present study, the distribution was not at all smooth, and alpha waves from different

87 individuals were found to be in three large groups. The alpha frequencies in these groups were

88 around 8,10 , and $12 \mathrm{~Hz}$ (waves per second). The groups are very easy to miss. In a histogram

89 showing the distribution of individual alpha frequencies, the groups are visible only if the bin

90 width is narrow.

91 The existence of three alpha groups was predicted from an alpha hypothesis (Johannisson \&

92 Nilsson, 1996), and the present study was designed in such a way that it was possible to test if

93 specific alpha groups exist. The experiment is very simple but somewhat time-consuming

94 because a narrow bin width in a histogram requires a relatively large number of observations.

95 When the alpha frequencies from 200 individuals were included in the histogram, the pattern

96 with three large alpha groups became clearly visible.

97 The link between alpha waves and personality dimensions was explored further in the present

98 study. The new finding of distinct alpha groups has to be taken into account when studying how

99 the alpha waves are related to personality dimensions and temperament types. 
100 METHODS

101 Participants

102 There were 200 participants in the study, and to reach this number, 204 healthy individuals were

103 invited to take part. Approval was obtained from the Regional Ethics Board for University of

104 Göteborg (R627-97) and written informed consent was obtained from all participants. For

105 participants younger than 18 years, written informed assent was also obtained from a parent. The

106 age of the participants ranged from 15 to 70 years, with a mean age of 37.5 years and a standard

107 deviation of 13.0 years. The study included 63\% females and $37 \%$ males. Of the participants,

$10834 \%$ were students, $26 \%$ nurses, $14 \%$ doctors, $7 \%$ social workers, and $19 \%$ were individuals

109 with other occupations.

110 EEG measurements

111 The individual alpha frequency was measured from single sequences of alpha waves where every

112 wave could be identified (Fig. 1). When testing the alpha hypothesis (Johannisson \& Nilsson,

113 1996), it is the actual wavelength that is of interest, rather than the amount of oscillatory

114 components in different frequency bands. For the purpose of this study, measurements directly

115 from sequences of alpha waves have a much higher accuracy compared to data from averaging

116 procedures using the Fourier transformation. A comparison between the two methods is made in

117 the Discussion section.

118

Figure 1

119 Figure 1 shows how the wavelength and alpha frequency were calculated. The number of waves

120 in a sequence of alpha waves divided by the time for the whole sequence gave the alpha

121 frequency. One sequence of alpha waves was used for each participant, and to ensure a

122 reasonably high degree of objectivity, the longest sequence was selected. For most of the

123 recordings, this procedure left very little room for subjective judgments. Sequences of alpha

124 waves from all participants are available in a supplemental file (Data S1).

125 Spontaneous activity was recorded while the participants rested in a reclining chair with their

126 eyes closed. Recording took place in a silent room with reduced lighting. The recording

127 conditions were optimized to enable the collection of clear and clean alpha waves. The

128 electrodes were placed on hairless areas of skin behind the ears and on the midline just below the

129 hairline. This made it possible to use tape electrodes that resulted in a considerably lower degree

130 of discomfort compared to ordinary arrangements. Moreover, the probability of getting long

131 sequences of alpha waves was expected to increase when the participant could relax during the

132 recording session. This also ensured that muscle activity seldom interfered with the recording. A

133 small amount of conductive gel was used and the impedance values were below $3 \mathrm{kOhm}$ for

134 most participants. 
135 Data were generally collected during a total time of 2.5 minutes (30 epochs of $5 \mathrm{~s}$ each, as shown

136 in Fig. 1). When there was a large amount of irregular activity, the recording time was prolonged

137 to increase the chance of obtaining a long sequence of alpha waves. Two channels were used,

138 corresponding to AFz-TP9 and AFz-TP10.

139 In a pilot study with EEGs from 213 subjects, different recording sites were compared in a 140 methodical way (unpublished data). A few recording electrodes were found to be sufficient to 141 measure the alpha frequency because alpha waves are widespread. For instance, the longest

142 sequence of regular waves was found in the occipital region in approximately half of the

143 recordings, and in $96 \%$ of these recordings, the same alpha waves were also seen in other

144 regions. When the longest sequence appeared in a non-occipital region, 88\% of the EEGs had the 145 same alpha waves recorded simultaneously from occipital electrodes.

146 In histograms showing the distribution of individual alpha frequencies, distinct groups are visible 147 only if the bin width is narrow. This requires a fairly high number of participants. In the pilot 148 study, various combinations of bin width and number of individuals were tested. A clear pattern 149 in the histogram required recordings from at least 200 subjects.

\section{Personality measurements}

151 The IPIP-NEO inventory was derived from the International Personality Item Pool

152 (http://www.ipip.ori.org). The 120-item version of the IPIP-NEO (Johnson, 2014) was used, and

153 all participants completed all items. An online analysis gave estimates with respect to five broad

154 domains and 30 subdomains of personality (http://www.personal.psu.edu/j5j/IPIP). After

155 adjusting for age and gender, the scores from the questionnaire were converted into percentile

156 estimates.

157 Compared with the reference data used in the online analysis, the participants in the present

158 study had, on average, higher extraversion scores and lower neuroticism scores. Moreover, many

159 of the participants in the present study had high scores in the trust and cooperation subdomains.

160 The differences described above may reflect an unintentional bias in the recruitment of

161 participants for this study. To facilitate comparison with future studies, the estimates are not

162 normalized to fit the total mean values in the present sample. The data from the personality

163 measurements are provided in a supplemental file (Data S2). 


\section{RESULTS}

165 The degree of extraversion and neuroticism is plotted against the individual alpha frequency in Fig. 2. The correlation lines in this figure are the usual way of describing the relationship between personality traits and alpha waves. However, there is another better way if we take into consideration that specific groups of alpha waves may exist.

Figure 2

\section{Three main alpha groups}

171 The histogram in Fig. 3A shows the distribution of alpha frequencies from different individuals.

172 The distribution of the data is not smooth and large groups are visible. The center of one group is

173 slightly above $8 \mathrm{~Hz}$ and the center of another group is somewhat below $12 \mathrm{~Hz}$.

Figure 3

175 The data ranging from 9 to $11 \mathrm{~Hz}$ can be described as one large group with a bimodal

176 distribution. A subdivision of the main group around $10 \mathrm{~Hz}$ gives a total of four groups, where

177 the subgroups have alpha frequencies around 9.5 and $10.5 \mathrm{~Hz}$.

178 Many participants did not have long sequences of well developed alpha waves (details can be found in a supplemental EEG file, Data S1). To test whether the existence of the alpha groups depends on how well the alpha waves are developed, the recordings were classified into three types: poorly, intermediately, and well developed alpha waves.

182 When the alpha waves are poorly developed, measurements of the individual alpha frequency

183 become problematic. All such measurements are excluded from Fig. 3B, and yet the three main

184 alpha groups and the bimodal distribution for the middle group remain.

185 When the participants with an intermediate degree of alpha waves are also excluded, leaving 186 only recordings with well developed alpha waves, as shown in Fig. 3C, the group around $12 \mathrm{~Hz}$ 187 becomes small and the bimodal distribution for the middle group is not visible. What remains are

188 a distinct group at $8 \mathrm{~Hz}$ and a gap at $9 \mathrm{~Hz}$. Relatively few participants are included in Fig. 3C, 189 and this histogram illustrates the problem with a narrow bin width and a small number of 190 subjects. 
191 Three alpha segments

192 In Fig. 4, the three main alpha groups are studied one at a time. Furthermore, mean values for the

193 personality estimates are used, with these mean values reducing the number of data points. For

194 example, there are four data points at $7.9 \mathrm{~Hz}$ in Fig. 2A and the mean value of the estimates from 195 these four participants is shown as one data point in Fig. 4A.

The relationship between the alpha frequency and personality dimensions is much better described by curved lines than by straight lines (Fig. 4). The group around $8 \mathrm{~Hz}$ has a high degree of extraversion at the center of the group but not in the surrounding zones (Fig. 4A). This pattern is also seen for the groups around 10 and $12 \mathrm{~Hz}$ (Fig. 4B-C).

The curves for neuroticism (Fig. 4D-F) indicate that there is a difference between the centers of the groups and the surrounding zones. Overall, Fig. 4 supports the existence of three main alpha groups and substantiates the notion that extraversion is high and neuroticism is low in the center of each group.

\section{Broad domains}

\section{Center versus surrounding area}

To test whether there was a statistically significant difference between the center and the surrounding area, the data from the surrounding zones in Fig. 4 were combined. The selected ranges were $7.05-7.65,8.55-9.35,10.65-11.35$, and $12.15-12.55 \mathrm{~Hz}$, and the sample size for the surrounding data was $n=43$. The ranges for the center data were 7.85-8.15, 9.85-10.15, and

$21111.65-11.95 \mathrm{~Hz}$, which resulted in a sample size of $n=49$. Two-tailed $t$-tests were used to 212 determine differences.

213 For extraversion, the difference between the center and the surrounding area was highly

214 significant $(p<0.001)$. The difference was also statistically significant for neuroticism $(p<0.01)$

215 and agreeableness $(p<0.05)$, but not for conscientiousness. For openness to experience, the

216 mean estimates were about the same for the center and the surrounding area (Fig. 5A). 


\section{Three alpha groups}

219 Figure 5B shows a comparison among the mean estimates for three groups of alpha waves. To

220 ensure an adequate sample size when the three groups were considered separately, wide ranges

221 for the center data were used $(7.75-8.45,9.45-10.55$, and $11.45-12.05 \mathrm{~Hz})$. The sample sizes of

222 these three groups were 34,68 , and 28.

223 The differences among the three groups in Fig. 5B were relatively small, but there were some

224 interesting trends. The group at $12 \mathrm{~Hz}$ had the highest score for all domains except for

225 extraversion. For openness to experience, the difference between the groups at 8 and $12 \mathrm{~Hz}$ was

226 statistically significant $(p<0.05)$.

\section{Four alpha groups}

228 The four groups shown in Fig. 5C had ranges of 7.95-8.35, 9.35-9.85, 10.15-10.65, and 11.45-

$22912.05 \mathrm{~Hz}$. These limits gave sample sizes of $26,33,34$, and 28 . The ranges for the four groups

230 were sufficiently narrow when searching for personality traits that are representative of the

231 centers of the alpha groups.

232 Figure 5C shows how the data were transformed after the division of the main group around 10

$233 \mathrm{~Hz}$ into subgroups around 9.5 and $10.5 \mathrm{~Hz}$. For neuroticism, the group at $9.5 \mathrm{~Hz}$ had the lowest

234 score and the group at $10.5 \mathrm{~Hz}$ had the highest score of the four groups. For agreeableness, the

235 group around $10.5 \mathrm{~Hz}$ stood out among the other groups.

236 The group at $9.5 \mathrm{~Hz}$ had the highest score on conscientiousness, but the score was just slightly

237 higher than that obtained from the group at $12 \mathrm{~Hz}$. The latter group still had the highest score on

238 openness to experience and the lowest score on extraversion.

\section{Subdomains}

240 Each of the five broad domains has six subdomains (Table 1). Most of the differences among the 241 four alpha groups were small, but when taken together they corresponded to four different

242 personality profiles.

Table 1

2448 waves per second - Friendly and impulsive

245 The group around $8 \mathrm{~Hz}$ had a very high score in the friendliness subdomain. This score was

246 significantly higher compared to the score for the group at $12 \mathrm{~Hz}(p<0.02)$. The group at $8 \mathrm{~Hz}$

247 also had a mean score that was clearly higher than that of the group at $10.5 \mathrm{~Hz}(p<0.02)$. The

248 difference was smaller when the group at $8 \mathrm{~Hz}$ was compared with the group at $9.5 \mathrm{~Hz}$, although

249 it was still statistically significant $(p<0.05)$. 
250 There were several other subdomains that could be used to characterize the group at $8 \mathrm{~Hz}$. In the 251 cautiousness subdomain, the group around $8 \mathrm{~Hz}$ had a lower mean score than did the groups 252 around $9.5 \mathrm{~Hz}(p<0.05)$ and around $12 \mathrm{~Hz}(p<0.05)$. The difference between the group at $8 \mathrm{~Hz}$ 253 and the group at $10.5 \mathrm{~Hz}$ was not large enough to be statistically significant.

254

255

256

257

258

259

260

261

262

263

264

265

266

267

268

269

270

271

\section{1/2 waves per second - Peaceful and reliable}

In the anger subdomain, the group around $9.5 \mathrm{~Hz}$ had the lowest score of the four groups. The difference was largest when compared with the group at $8 \mathrm{~Hz}$, although this result did not reach statistical significance.

The group at $9.5 \mathrm{~Hz}$ had relatively high estimates in almost all of the conscientiousness subdomains. In the dutifulness subdomain, this group had a higher score than the group around 8 $\mathrm{Hz}$, and this difference was statistically significant $(p<0.05)$. Also, when compared with the groups at 10.5 and $12 \mathrm{~Hz}$, the group at $9.5 \mathrm{~Hz}$ had a higher mean score, although these differences were not statistically significant.

\section{$10^{1 / 2}$ waves per second - Worried and less agreeable}

People in the group at $10.5 \mathrm{~Hz}$ had a mean score in the anxiety subdomain that was higher than that in the groups at $9.5 \mathrm{~Hz}(p<0.05)$ and at $12 \mathrm{~Hz}(p<0.05)$. When compared with the group at $8 \mathrm{~Hz}$, the group at $10.5 \mathrm{~Hz}$ had a somewhat higher score, but the difference was not statistically significant.

In the agreeableness subdomains, while the group at $10.5 \mathrm{~Hz}$ did not have low scores, the other three groups generally exhibited scores that were slightly higher. The difference between the group around $10.5 \mathrm{~Hz}$ and the group around $12 \mathrm{~Hz}$ in the cooperation subdomain was statistically significant $(p<0.05)$.

\section{2 waves per second - Lesshappy and more open}

The group at $12 \mathrm{~Hz}$ had a somewhat lower score on cheerfulness and a slightly higher score in the depression subdomain than the other groups, but these differences were not large enough to be statistically significant. There was a tendency for the group at $12 \mathrm{~Hz}$ to have a more open cognitive style compared to the other three groups. The subdomains associated with openness to experience in Table 1 provide more detailed information than does the broad domain shown in Fig. 5C. 


\section{Happiness}

280 The cheerfulness, anxiety, and depression subdomains are all related to happiness. The three

281 curves in Fig. 6A indicate that a high degree of happiness can be found in the center of the group

282 around $8 \mathrm{~Hz}$.

Figure 6

284 The main group around $10 \mathrm{~Hz}$ had a reasonably high degree of happiness in a frequency range

285 that was much wider than the ranges for the groups around 8 and $12 \mathrm{~Hz}$ (Fig. 6). Thus, there was

286 sufficient space for the subgroups at 9.5 and $10.5 \mathrm{~Hz}$.

287 People in the main group at $10 \mathrm{~Hz}$ had a curve for depression that was shifted slightly to the right

288 when compared with the curve for anxiety (Fig. 6B). This was also seen for people in the group

289 at $8 \mathrm{~Hz}$ (Fig. 6A).

290 In the cheerfulness subdomain, the maximum level for the group at $12 \mathrm{~Hz}$ was somewhat lower

291 compared to that for the other groups (Fig. 6). The line for depression in the group at $12 \mathrm{~Hz} \mathrm{did}$

292 not have a bend in the same direction as the other groups (Fig. 6). When compared with those in

293 the center of the group at $12 \mathrm{~Hz}$, people in the surrounding zones had much higher anxiety scores

294 (Fig. 6C).

\section{DISCUSSION}

296 The primary aim of the present study was to test the two levels hypothesis (Johannisson, 1984).

297 This hypothesis deals with the general organization of the forebrain, and, if it withstands

298 repeated testing, it may contribute to our understanding of the relationship between mental

299 activity and brain activity.

\section{The two levels hypothesis}

301 In the model, a few cerebral columns have activity at a high level at each moment in time. The

302 high-level activity is short-lasting and a column has activity at a comparatively low level almost

303 all the time (Johannisson, 1984).

304 The two levels of activity are separate because between them, there is a gap in the activity range

305 (Johannisson, 1993). In this context, activity at a high level in the columns is activity at a

306 conscious level. When this activity is observed from outside, it is seen as neuronal activity, and

307 when the same activity is observed from inside, it is seen as mental activity. 
308 Each column has a set of connections with other columns and other parts of the nervous system.

309 These connections constitute the functional properties that characterize every single column

310 (Johannisson, 1984). The various connections are of different strengths, and association paths in

311 cognitive processes usually follow the strongest connections. However, weak outgoing

312 connections from two or more columns with high activity may converge on a new column and

313 produce additive effects, such that they can sometimes be stronger together than a strong

314 connection from a single column (Johannisson, 1984; Johannisson, 1993). The association path

315 then takes another direction rather than simply following the most obvious connection.

316 A direct recording of high neuronal activity should be possible if a recording electrode is placed 317 close to a cerebral column. However, high activity in a column is a rare event because there are

318 very many columns. This direct approach is also problematic because a recording of high activity

319 may be difficult to repeat. Moreover, if high activity is recorded, it has to be distinguished from

320 other types of high activity, such as that caused by damage from the recording electrode.

321 Alpha waves are not direct recordings of high activity in the cerebral columns. On the contrary,

322 alpha waves are thought to represent neuronal activity at the low level, albeit from many cerebral

323 columns (Johannisson \& Nilsson, 1996). Small but synchronous changes up and down in the

324 neuronal activity within the low activity range produce the alpha waves in this model.

325 Nonetheless, the alpha waves reflect the regulating signals that keep the number of highly active 326 columns within proper limits.

327 The two levels hypothesis is falsifiable. In this paper, the focus is on the existence of specific

328 alpha groups, because without them the hypothesis cannot be true. At the outset of the study, the

329 existence of these groups seemed unlikely because the prevailing opinion is that the distribution

330 of individual alpha frequencies is bell-shaped and without gaps (Klimesch, 1999; Robinson,

331 2006; Fink \& Neubauer, 2008; Başar, 2012; Bazanova \& Vernon, 2014). The present study had

332 to include measurements from a relatively large number of individuals before the alpha groups

333 could be clearly seen (Fig. 3A).

\section{Testing}

335 The alpha groups are accessible for rigorous testing. The initial finding of three distinct alpha 336 groups was made in a pilot study of 213 recordings from an EEG clinic (unpublished data). The 337 result was confirmed in the present study with an entirely different sample. 
338 The individual alpha frequency can be measured in different ways (Başar, 2012; Bazanova \& 339 Vernon, 2014). Any method can be used to test the existence of the alpha groups as long as it 340 is sufficiently precise. Measurements from a power spectrum with very narrow bands ought to 341 give results that are comparable to those based on measurements from long sequences of 342 alpha waves, at least when the alpha waves are well developed.

343 A major problem with the power spectrum and other averaging procedures is that they include 344 a large amount of uncertain data. For testing the alpha hypothesis, only sequences with 345 regular alpha waves are relevant (Johannisson \& Nilsson, 1996). Irregular activity and various 346 artifacts should not be included.

347 Another and more serious problem with measurements based on averaging procedures is that 348 they are affected by the amplitude. Long waves often have higher amplitude compared to 349 short waves, and waves with high amplitude weight the average to a greater extent than do 350 small waves. This causes a large systematic error in frequency measurements based on 351 averaging procedures. Inaccuracy of this type is not a problem when using sequences of alpha 352 waves, as in the present study, because frequency measurements from sequences of waves are 353 not dependent on the amplitude of the waves.

354 A method based on the power spectrum of alpha activity is not more objective than a method 355 using the longest wave sequences containing regular alpha waves. Both methods are easy to 356 use when there are well developed alpha waves. Thus, in a power spectrum, there can be a 357 clear peak, which simplifies the measurement of the alpha frequency. However, when the 358 alpha waves are poorly developed, the power spectrum often contains more than one peak, 359 and the peaks are usually broad and asymmetric. In such cases, it is not easy to determine how 360 the measurement should be done.

361 Thorough testing requires that independent researchers repeat the experiment in order to 362 confirm the results. When preparing for new studies, perhaps it can be of some help that all of 363 the alpha sequences behind the histograms in Fig. 3 are reproduced in a supplemental EEG 364 file (Data S1). This file shows how long the longest sequence is for every participant. The 365 second and third longest sequences are often included and they give an idea of the size of the

366 variation in the measurements, but only the longest sequence was used. Exactly how the alpha

367 frequency was calculated is shown for every participant. Also, the amount of noise and the 368 general quality of the recordings can be assessed from this file. 


\section{Four temperaments}

370 A bimodal distribution of the alpha frequencies in the main group around $10 \mathrm{~Hz}$ was found in

371 the present study (Fig. 3A-B). The two subgroups were not predicted from the alpha

372 hypothesis and they were not visible in the pilot study. Therefore, the finding of a bimodal

373 distribution was not expected.

374 In the pilot study, most of the subjects were patients taking medication that might affect their

375 alpha waves. There were no such complications in the present study, and this may explain why

376 the shallow valley in the middle of the large group around $10 \mathrm{~Hz}$ can be seen in the present study but not in the pilot study. A re-examination of the recordings from the subgroups around 9.5 and $10.5 \mathrm{~Hz}$ confirmed the bimodal distribution in the present study.

The personality profiles characterized in Table 1 and Fig. 6 can be recognized as four types of temperament that resemble the four classical temperaments. Many scientists consider the classical temperaments to be obsolete, and though there are many descriptions of these temperaments, there are no useful definitions. Thus, it is not possible to test whether the four profiles found in the present study are identical to the four classical temperaments.

384 It has previously been suggested that the definitions of the four classical temperaments use a combination of two personality dimensions (Robinson, 2001). This method of defining the temperaments is very elegant, but it does not take into account that the main differences for extraversion and neuroticism were found when the centers of the groups were compared with the surrounding zones (Fig. 4 and 5A). For example, if the sanguine temperament is defined as having high extraversion and low neuroticism (Robinson, 2001), then people from the centers of all four groups would be included (Fig. 4). However, this idea is not altogether ineffective because extraversion was slightly higher in the group at $8 \mathrm{~Hz}$ compared to the other three groups, and neuroticism was slightly lower in the group at $8 \mathrm{~Hz}$ than it was in two of the other three groups (Fig. 5C).

394 As another example, consider the case in which the melancholic temperament is defined as having low extraversion and high neuroticism (Robinson, 2001). In the present study, extraversion was slightly lower in the group at $12 \mathrm{~Hz}$ compared to the other three groups, while neuroticism was slightly higher in the group at $12 \mathrm{~Hz}$ than it was in two of the other three groups (Fig. 5C). Thus, the definition of the melancholic temperament based on two personality dimensions has some support when the comparison is made among the four groups. The problem with this definition comes to light when people from the surrounding zones are

401 included, because they usually have low scores on extraversion and high scores on neuroticism 402 (Fig. 5A). 
403 Instead of having a parallel terminology with new names (such as $8,9 \frac{1}{2}, 10 \frac{1}{2}$, and 12 ), it is

404 proposed that the temperaments be defined by the alpha frequency. The sanguine temperament

405 would then refer to the group around $8 \mathrm{~Hz}$, the phlegmatic temperament to the group at $9.5 \mathrm{~Hz}$,

406 the choleric temperament to the group at $10.5 \mathrm{~Hz}$, and the melancholic temperament to the group

407 around $12 \mathrm{~Hz}$.

408 The four temperaments refer to the centers of the alpha groups. Both the centers and the

409 surrounding areas should be included when the alpha waves are correlated to personality traits.

410 Thus, the best description of the link between alpha waves and personality traits is provided by

411 the curved lines in Fig. 4 and 6.

412 An attempt is made in the remaining part of the Discussion section to explain the nature of the

413 underlying relationship between alpha waves and personality traits. The two levels hypothesis

414 (Johannisson, 1984; Johannisson, 1993) and the subsequent alpha hypothesis (Johannisson \&

415 Nilsson, 1996) are applied fully, and their implications in various fields are discussed.

\section{Number of highly active columns}

417 When the alpha frequency is $10 \mathrm{~Hz}$, it has been estimated that the number of highly active

418 columns alternates between 4 and 5 (Johannisson \& Nilsson, 1996). The large group at $8 \mathrm{~Hz}$ has

419 alpha waves that appear when the number of columns is switching between 3 and 4 (Johannisson

420 \& Nilsson, 1996; Johannisson, 1997). In a similar way, the group at $12 \mathrm{~Hz}$ is formed by alpha

421 waves that are generated when the number of columns at the high level is alternating between 5

422 and 6 (Johannisson \& Nilsson, 1996; Johannisson, 1997).

423 In addition to the alpha groups, there are other frequency groups. Infants exhibit waves that have 424 a frequency of around $4 \mathrm{~Hz}$, which may appear when the number of highly active columns is 425 alternating between 1 and 2 (Johannisson, 1997). The number may increase with age, and when 426 it alternates between 2 and 3, the waves become shorter and form a group around $6 \mathrm{~Hz}$. If the 427 increase in number continues, the limits become 3 and 4, and the frequency group is at $8 \mathrm{~Hz}$.

428 Some individuals stay within this sanguine group, while for others, the limits are raised further to $4294-5$ or 5-6. When awake, very few individuals have the limits 6 and 7 , which give a frequency 430 of around $14 \mathrm{~Hz}$.

431 During the different sleep stages, all of the frequency groups described above may appear 432 (Johannisson, 1997). Moreover, a frequency around $1 \mathrm{~Hz}$ may appear when the limits are 0 and 1 433 during deep or very deep sleep. Thus, adults have regulating systems that retain the capacity to 434 keep the number of highly active columns within several different limits. 


\section{Regulating system}

436 When the activity in a column jumps up to the high level, the upper limit for the number of

437 highly active columns is reached. The regulating system has to react quickly to decrease the

438 excitability of all of the columns with low activity (Johannisson \& Nilsson, 1996). If there is a

439 delay, additional columns can change their activity from the low to the high level and the

440 number will exceed the upper limit.

441 Occasionally, two new columns may change the activity from the low to the high level at the

442 same time. When this occurs, the number will exceed the upper limit. At this point, the

443 regulating system has to decrease the excitability of the remaining columns and wait until two

444 columns have stopped exhibiting high-level activity. If the regulating system fails and

445 increases the excitability already when the activity in only one column has left the high level,

446 the system can become trapped in repeated attempts to adjust the number. Strong regulating

447 signals that are repeated with very short intervals damage the thinking processes and may

448 explain what happens during a grand mal seizure.

449 The regulating system has to be very sensitive and react extremely quickly, which may lead to 450 mistakes. If a decrease in excitability starts before a new column has changed the activity 451 from the low to the high level, the number can go below the lower limit. The implication from 452 this reasoning might be that in a grand mal seizure, the number of highly active columns is 453 alternating between the upper limit and one step higher, while in a petit mal seizure, the 454 number is alternating between the lower limit and one step lower.

455 However, under normal conditions, the number is kept within proper limits. This number is

456 the first of two basic parameters in the model, and the second is the duration of high-level

457 activity of a column (Johannisson \& Nilsson, 1996).

\section{Different durations}

459 The limits for the number of highly active columns are the same in the groups around 9.5 and $46010.5 \mathrm{~Hz}$. The difference between these groups lies in the duration of high-level activity. In other 461 words, different durations may account for the underlying difference between people with a 462 phlegmatic temperament and those with a choleric temperament.

463 The alpha hypothesis makes it possible to calculate the duration of high-level activity in a 464 column (Johannisson \& Nilsson, 1996). The duration is the mean number of highly active 465 columns times the wavelength. As an example, the mean number is 4.5 when the number of 466 highly active columns is alternating between 4 and 5 . The wavelength for an alpha wave is not 467 the usual spatial period, but a temporal period, and therefore the inverse of the alpha frequency. 468 For example, if the frequency is $10.0 \mathrm{~Hz}$, then 1 divided by 10.0 /s gives the wavelength $100 \mathrm{~ms}$.

469 From these data, the duration of the activity at the high level in a column can be calculated as 4.5 $470 \times 100 \mathrm{~ms}=450 \mathrm{~ms}$. 
471 When the frequency is $9.5 \mathrm{~Hz}$, the duration is approximately $4.5 \times 105 \mathrm{~ms} \approx 470 \mathrm{~ms}$. In the same 472 way, a frequency of $10.5 \mathrm{~Hz}$ gives the duration $\approx 4.5 \times 95 \mathrm{~ms} \approx 430 \mathrm{~ms}$. Differences in the

473 duration thus account for the spreading of the frequency within the alpha groups.

474 Some individuals have alpha frequencies outside the main groups, for example, around 9 or 11

$475 \mathrm{~Hz}$. Although the computation is more complicated for alpha waves that are not within the main

476 groups, it is still possible to calculate both the number and the duration if there are two stable

477 rhythms available (Johannisson \& Nilsson, 1996). In EEGs, switching between two frequencies

478 is not uncommon, especially in young adults. Sufficient information is also available in sleep

479 EEGs because several rhythms appear during sleep.

480 It is important to be able to measure the duration of high-level activity in a column because this 481 duration determines the speed of the thinking processes. A relatively short duration, as seen in 482 the choleric group, designates a fast turnover of highly active columns and rapid thinking. The 483 opposite is seen in the phlegmatic group, where slow but reliable thinking processes are the 484 result of a long duration and a slow turnover.

485 Differences between cholerics and phlegmatics in the duration of the high activity of the columns 486 can explain the different mean scores observed in the cautiousness subdomain (Table 1). Short 487 duration and speedy thinking can lead to a high degree of impulsiveness.

488 In the anxiety subdomain, the phlegmatic group had a somewhat lower score than did the 489 choleric group (Table 1 and Fig. 6). This may be an effect of the relatively long duration in the 490 phlegmatic group.

\section{Number and duration together}

492 In this paper, an alpha hypothesis (Johannisson \& Nilsson, 1996) is used to explain the link 493 between alpha waves and personality traits. Starting from measurements of the alpha

494 frequencies, the two basic parameters in the hypothesis were calculated and the result is shown in 495 Fig. 7.

Figure 7

497 When the number of highly active columns alternates between 3 and 4, the duration of high-level

498 activity can be relatively short (Fig. 7). Conversely, when the number alternates between 5 and 6 , 499 the duration is comparatively long. A high number may give a flow of thoughts that can be 500 complicated. A prolonged duration makes it possible to handle complicated thoughts because 501 this duration results in slow but reliable thinking processes. 
502 The four temperaments are associated with four combinations of number and duration. These

503 combinations are shown as ellipses in Fig. 7. Outside the ellipses, in all directions, the values for

504 number and duration are suboptimal. Anxiety, depressed mood, and other symptoms may appear

505 in the surrounding zones (Fig. 6). Thus, the number-duration diagram opens up for a new

506 systematic way of understanding different mental disorders.

507 CONCLUSIONS

508 1. Alpha waves from different individuals can be categorized into three main groups where the

509 frequencies are around 8,10, and $12 \mathrm{~Hz}$. The middle group appears to have a bimodal

510 distribution, such that subdivision produces a total of four alpha groups.

511 2. When alpha waves are correlated with personality dimensions, the alpha frequency range is

512 better divided into three segments. In the centers of the main alpha groups, extraversion is high

513 and neuroticism is low.

514 3. There are many small differences in personality traits among the four groups. When taken

515 together, the groups comprise four personality profiles that are similar to the four classical

516 temperaments.

517 4. The link between alpha waves and personality traits (Fig. 8) implies an underlying

518 relationship. There are two hypotheses that can be applied. One of these deals with the general

519 organization of the forebrain and the other explains why alpha waves are generated.

Figure 8

\section{$521 \quad$ ACKNOWLEDGEMENT}

522 I would like to thank Jonatan Wistrand for the valuable discussions.

\section{SUPPLEMENTAL INFORMATION}

524 Data S1. Supplemental EEG File.

525 Data S2. Supplemental Personality File. 


\section{REFERENCES}

527 Başar E. 2012. A review of alpha activity in integrative brain function: Fundamental

528 physiology, sensory coding, cognition and pathology. International Journal of

529 Psychophysiology 86:1-24.

530 Bazanova OM, Vernon D. Interpreting EEG alpha activity. 2014. Neuroscience \&

531 Biobehavioral Reviews 44:94-110.

532 Fink A, Neubauer AC. 2008. Eysenck meets Martindale: The relationship between

533 extraversion and originality from the neuroscientific perspective. Personality and Individual

534 Differences 44:299-310.

535 Gale A. 1983. Electroencephalographic studies of extraversion-introversion: A case study in

536 the psychophysiology of individual differences. Personality and Individual Differences

537 4:371-380.

538 Hagemann D, Hewig J, Walter C, Schankin A, Danner D, Naumann E. 2009. Positive

539 evidence for Eysenck's arousal hypothesis: A combined EEG and MRI study with multiple

540 measurement occasions. Personality and Individual Differences 47:717-721.

541 International Personality Item Pool: A Scientific Collaboratory for the Development of

542 Advanced Measures of Personality and Other Individual Differences. Available at

543 http://www.ipip.ori.org (accessed 7 June 2016).

544 Johannisson T. 1984. Forebrain function: A theory about the general organization. Medical

545 Hypotheses 13:317-327.

546 Johannisson T. 1993. Cerebral cortex function: A general principle. Medical Hypotheses

$54740: 125-126$.

548 Johannisson T. 1997. Columnar analysis of sleep electroencephalogram. Medical Hypotheses

549 49:187-189.

550 Johannisson T, Nilsson H. 1996. The alpha rhythm in the EEG: A theory based on a 551 neurophysiological model. Medical Hypotheses 46:557-561.

552 Johnson JA. 2014. Measuring thirty facets of the Five Factor Model with a 120-item public

553 domain inventory: Development of the IPIP-NEO-120. Journal of Research in Personality

$554 \quad 51: 78-89$. 
555 Johnson JA. The IPIP-NEO. Available at http://www.personal.psu.edu/j5j/IPIP (accessed 7 556 June 2016).

557 Klimesch W. 1999. EEG alpha and theta oscillations reflect cognitive and memory performance:

558 A review and analysis. Brain Research Reviews 29:169-195.

559 Robinson DL. 2001. How brain arousal systems determine different temperament types and the 560 major dimensions of personality. Personality and Individual Differences 31:1233-1259.

561 Robinson DL. 2006. In pursuit of knowledge. International Journal of Psychophysiology $56262: 394-410$.

563 Tran Y, Craig A, McIssac P. 2001. Extraversion-introversion and 8-13 Hz waves in frontal 564 cortical regions. Personality and Individual Differences 30:205-215. 
Figure 1

Alpha waves.

(A) Short sequences of alpha waves. (B) An example of a sequence used in the present study. Blue tracings are from AFz-TP9 and red tracings are from AFz-TP10.
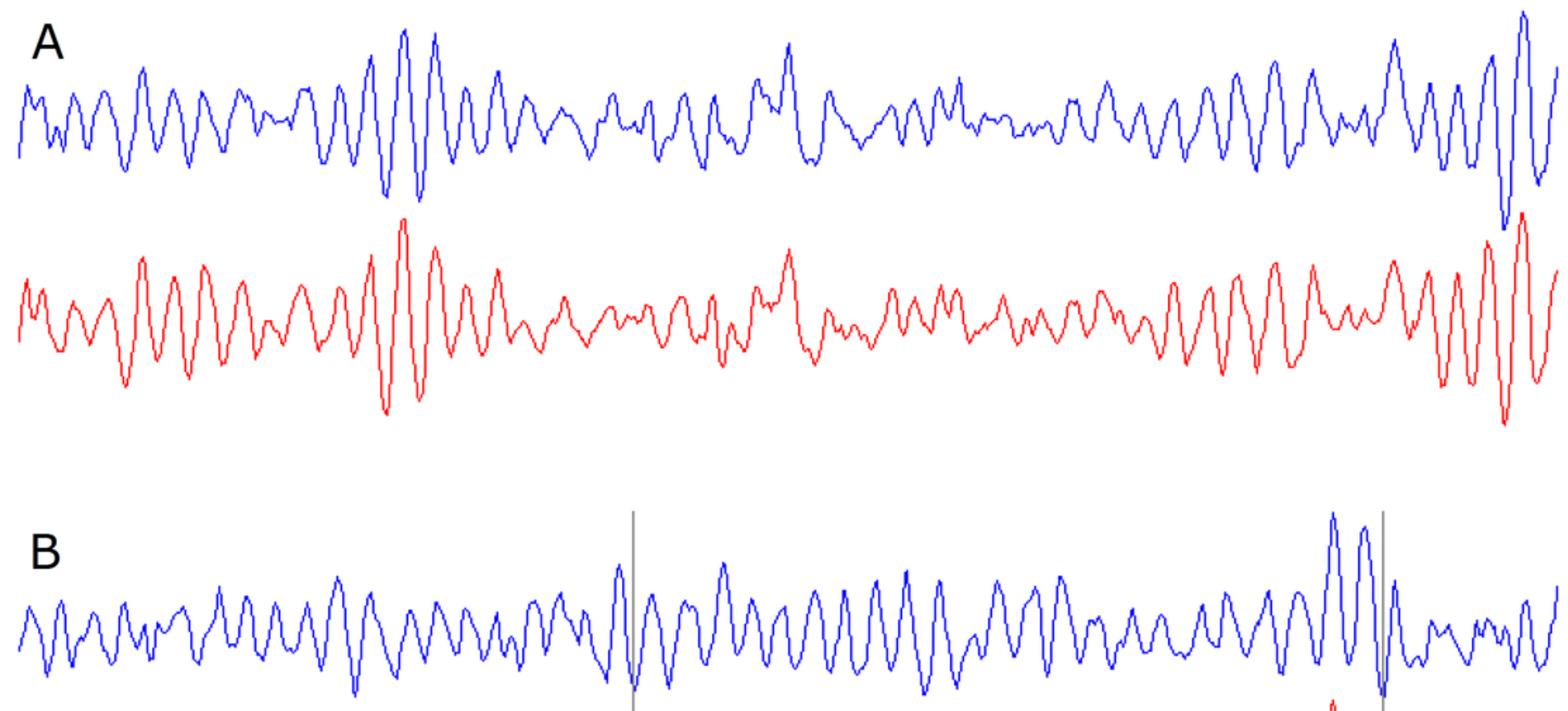

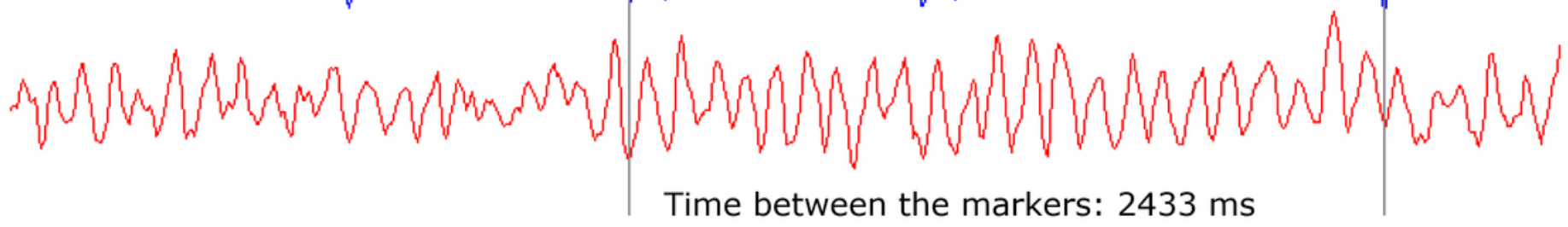
$\mid$\begin{tabular}{rr}
$20 \mu \mathrm{V}$ \\
$500 \mathrm{~ms}$ \\
\hline
\end{tabular} Number of waves between the markers: 23 Wavelength: $2433 \mathrm{~ms} / 23 \approx 105.8 \mathrm{~ms}$ Frequency: $1 / 105.8 \mathrm{~ms} \approx 9.5 / \mathrm{s}$ 
Figure 2

Scatter diagrams showing alpha frequency versus extraversion and neuroticism.

Data for all 200 participants in the present study. The correlation coefficient for extraversion was -0.16 (statistically significant, $p<0.05$ two tails) and the correlation coefficient for neuroticism was 0.02 (not statistically significant).
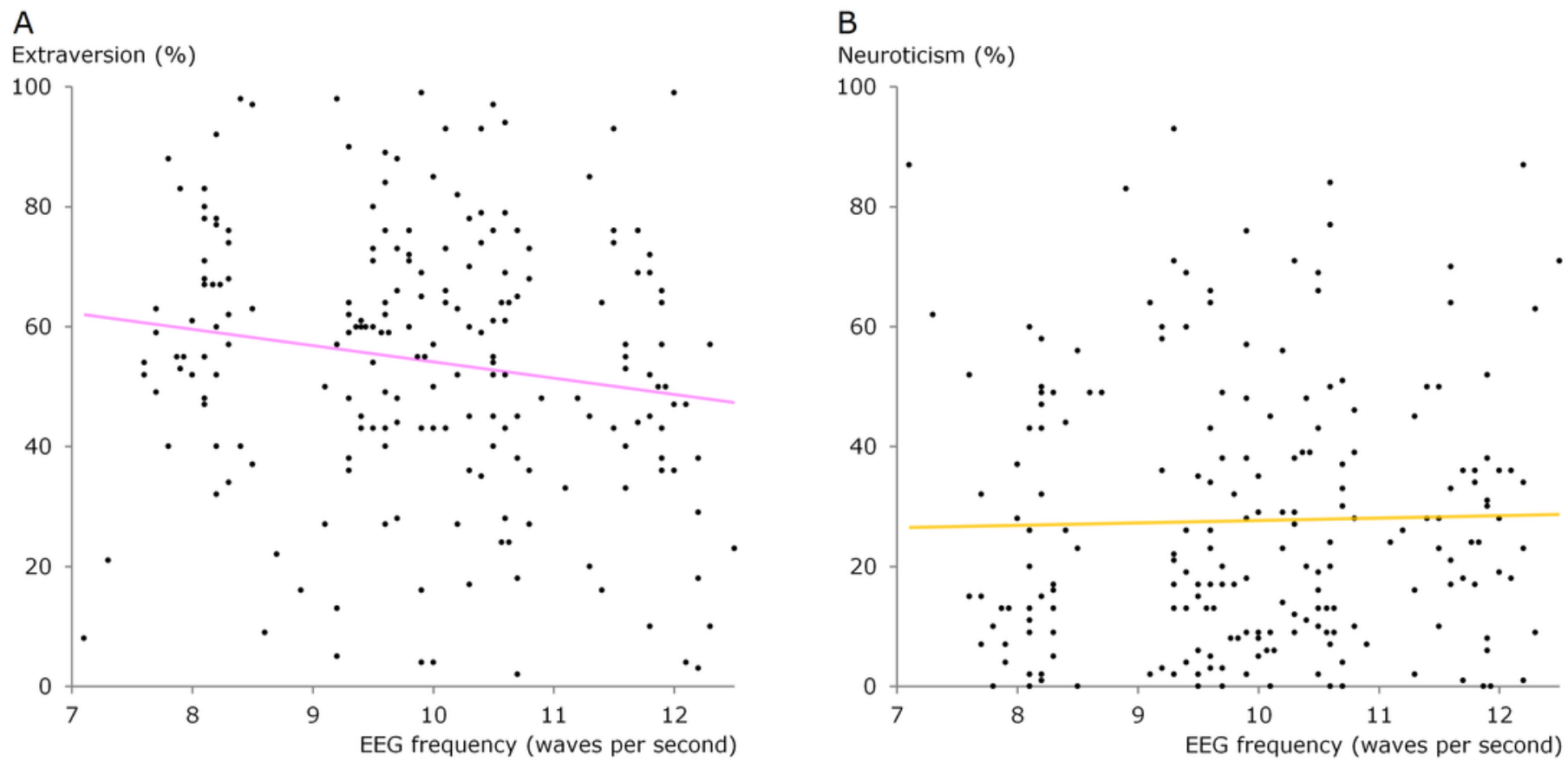
Figure 3

Distribution of individual alpha frequencies.

The histograms have a bin width of $0.1 \mathrm{~Hz}$. (A) All 200 participants. The recordings include poorly, intermediately, and well developed alpha waves. (B) As a control, recordings with poorly developed alpha waves are excluded. This reduced the sample size to $n=150$. (C) Only recordings with well developed alpha waves $(n=69)$.

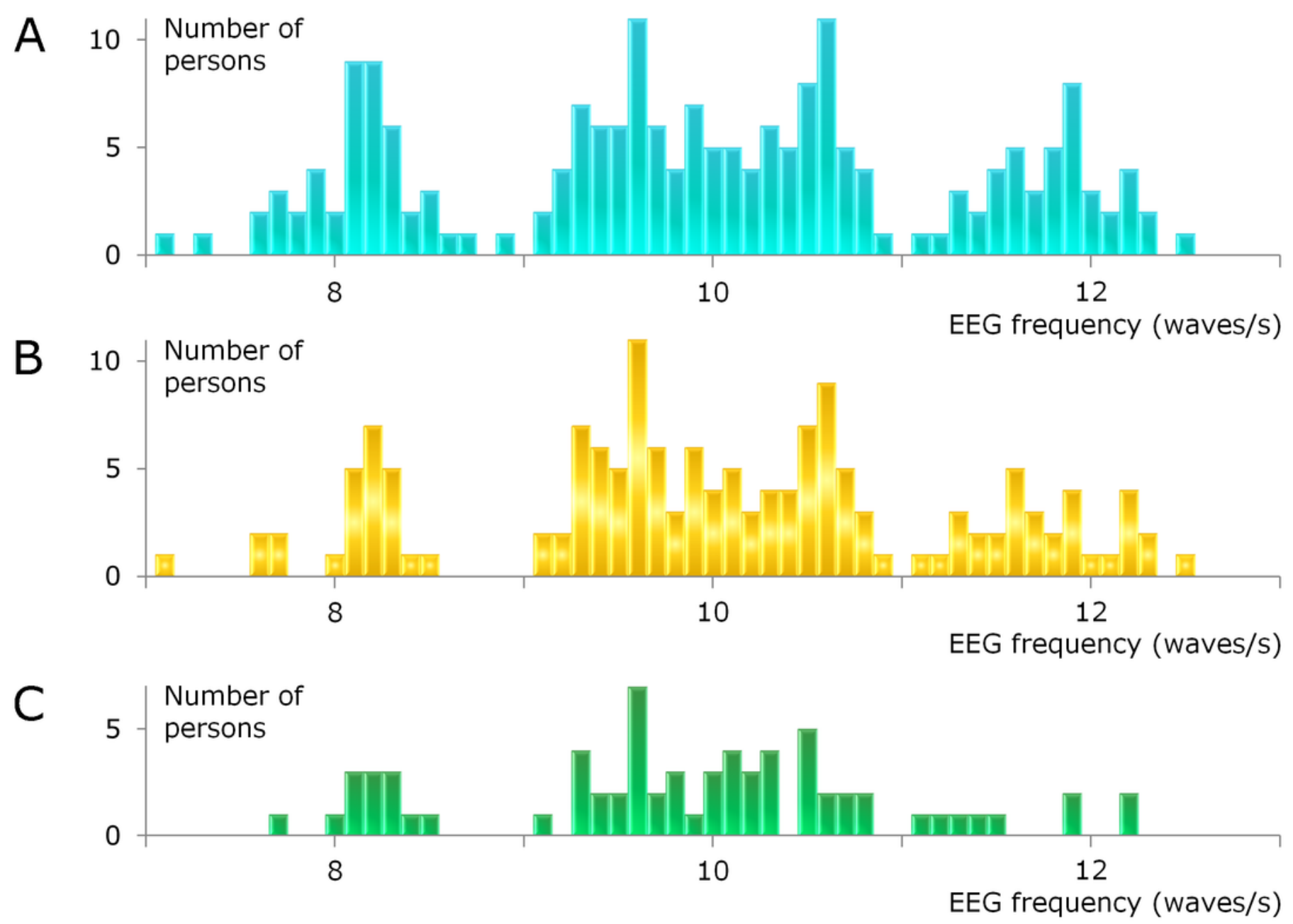




\section{Figure 4}

Three alpha segments.

The frequency range was divided into three segments and the personality estimates are shown as mean values. (A-C) Extraversion. (D-F) Neuroticism. The trend lines are secondorder polynomials.
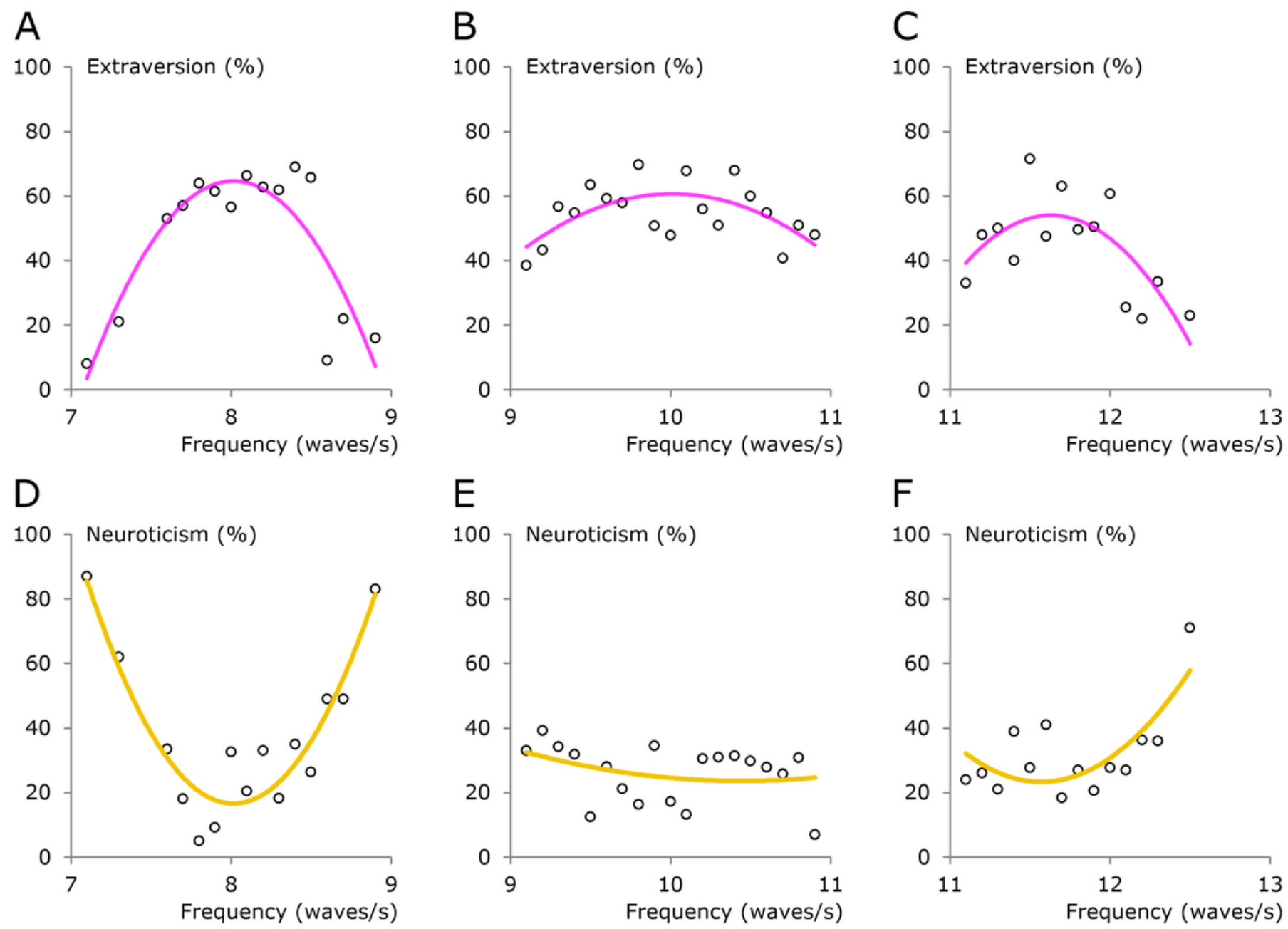
Figure 5

Mean estimates of personality traits in five domains.

(A) Center and surrounding area. (B) Three alpha groups. (C) Four alpha groups.
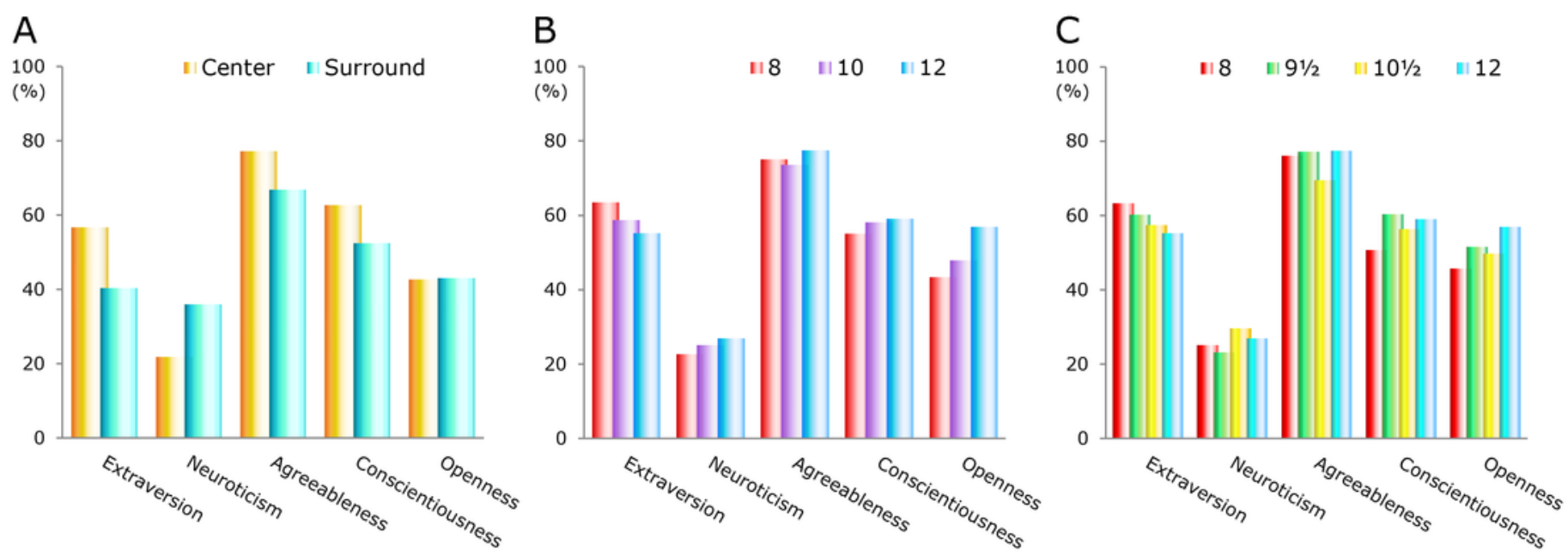
Figure 6

Curved lines representing estimates for the cheerfulness, anxiety, and depression subdomains.

The curves, which are second-order polynomials, were generated via polynomial regression. Light-colored rectangles denote four groups of alpha waves.
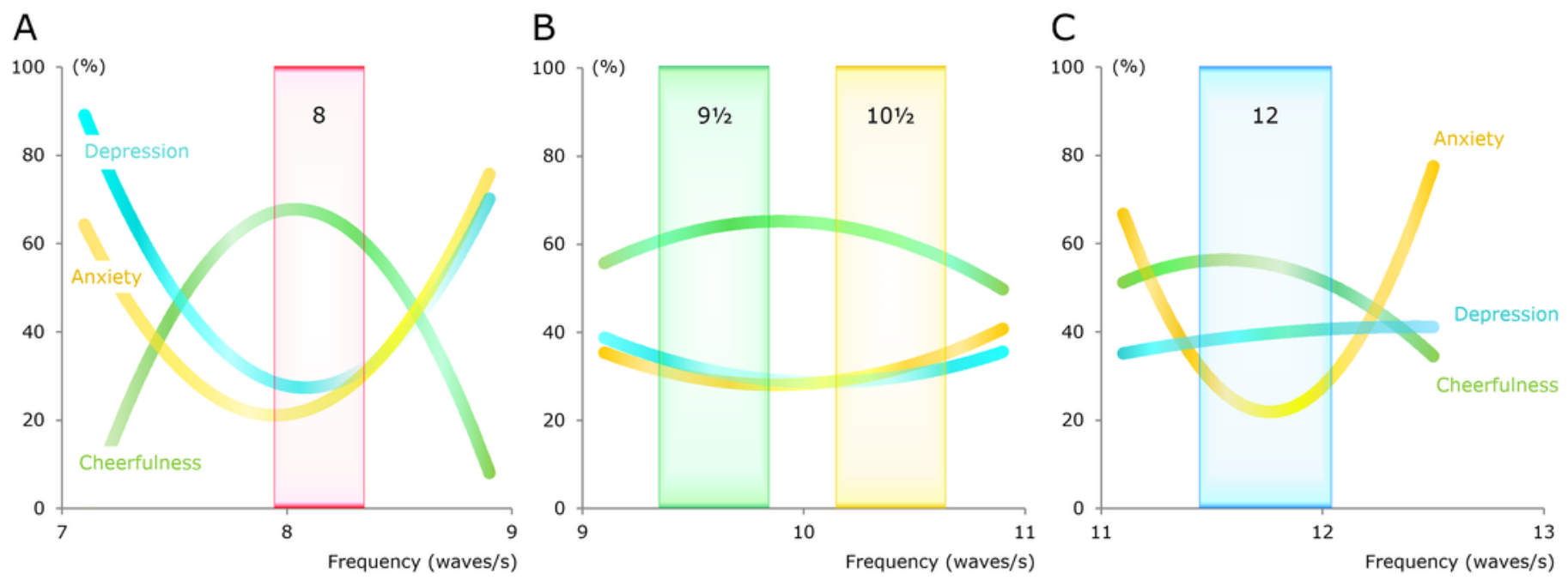


\section{Figure 7}

Number-duration diagram.

The $x$-axis shows the mean number of columns with activity at the high conscious level and the $y$-axis shows the mean duration of high-level activity in a column. One data point per participant. The ellipses indicate four types of temperament, and, as seen in the figure, the number and duration are somewhat different for these four. The sanguine temperament (in red) is characterized, in relative terms, by a low number and a short duration. Both the phlegmatic temperament (in green) and the choleric temperament (in yellow) have a medium number, but they have different durations. Furthermore, the melancholic temperament (in blue) can be seen as the result of having a high number and a long duration. 


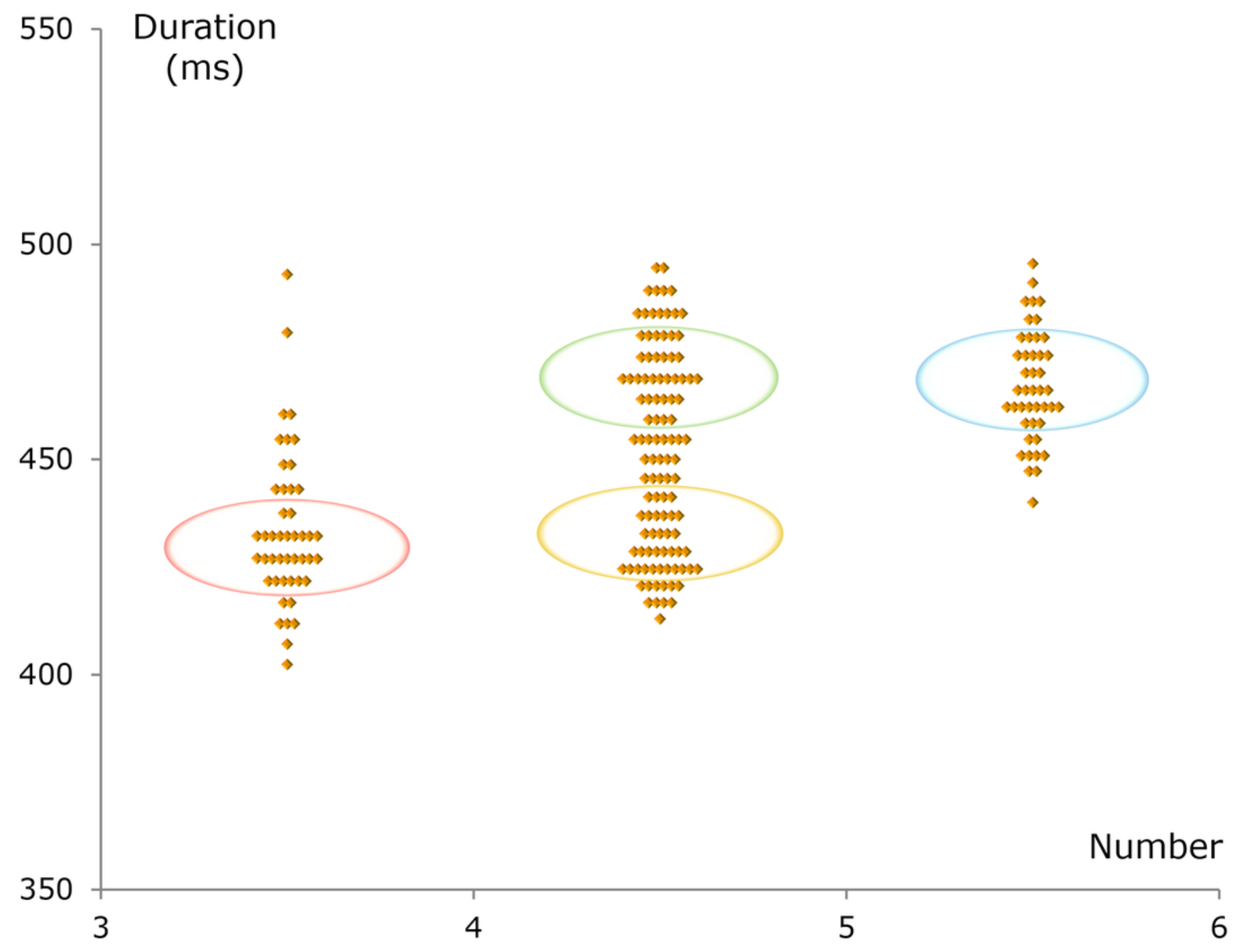


Figure 8

Summary illustrating how alpha waves, temperament types, and personality dimensions are interrelated.

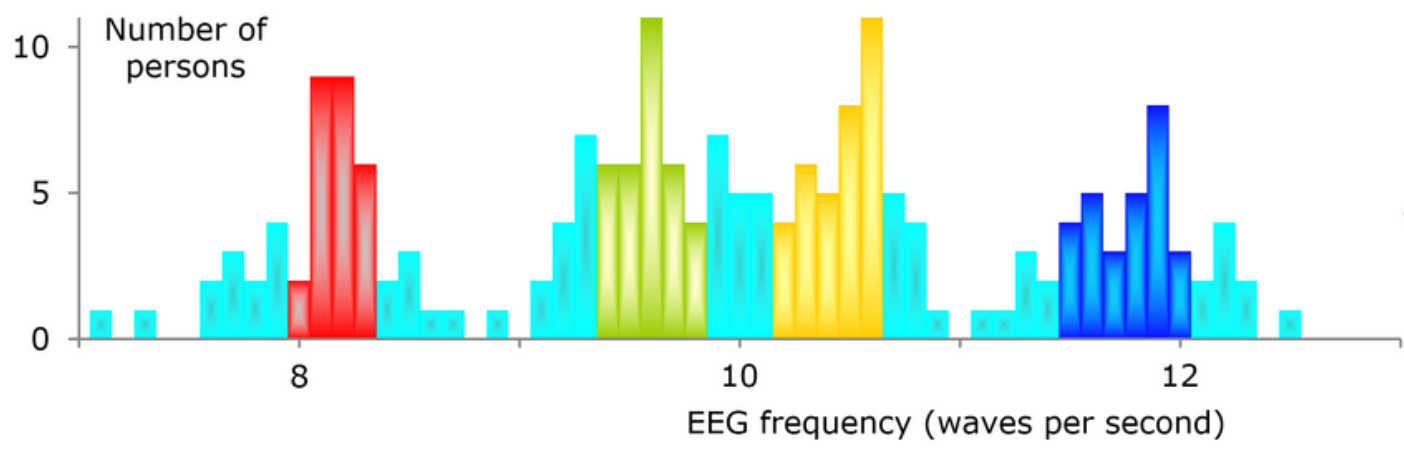

Extraversion (\%)

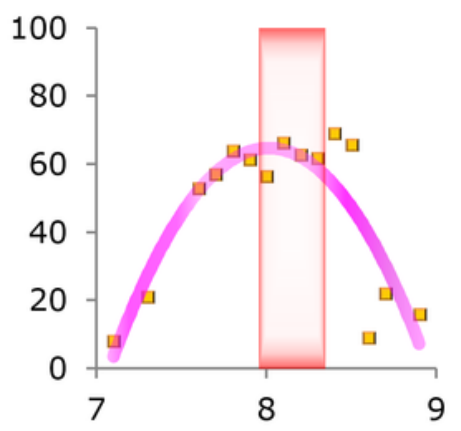

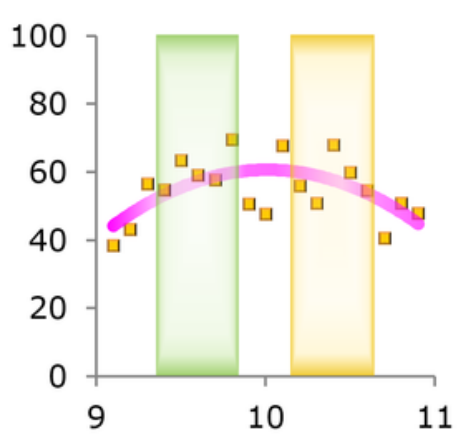

Four groups

of alpha waves

are linked to

temperament types

sanguine

phlegmatic

choleric

melancholic

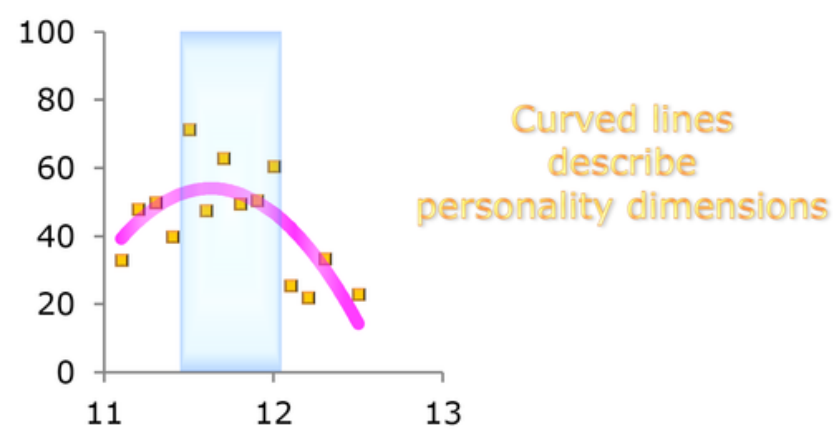




\section{Table $\mathbf{1}$ (on next page)}

Thirty subdomains from the five-factor model (Johnson, 2014).

The four alpha groups $(8,9.5,10.5$, and 12$)$ were defined by the alpha frequency in the same way as in Fig. 5C. 


\begin{tabular}{|c|c|c|c|c|c|}
\hline & & 8 & 9.5 & 10.5 & 12 \\
\hline \multirow[t]{6}{*}{ EXTRAVERSION } & Friendliness & 79 & 70 & 66 & 65 \\
\hline & Gregariousness & 79 & 68 & 69 & 76 \\
\hline & Assertiveness & 43 & 49 & 49 & 42 \\
\hline & Activity Level & 38 & 48 & 45 & 47 \\
\hline & Excitement-Seeking & 39 & 40 & 37 & 34 \\
\hline & Cheerfulness & 66 & 62 & 60 & 54 \\
\hline \multirow[t]{6}{*}{ NEUROTICISM } & Anxiety & 30 & 23 & 35 & 22 \\
\hline & Anger & 37 & 25 & 32 & 31 \\
\hline & Depression & 29 & 29 & 35 & 39 \\
\hline & Self-Consciousness & 25 & 37 & 32 & 34 \\
\hline & Immoderation & 34 & 36 & 40 & 36 \\
\hline & Vulnerability & 37 & 33 & 39 & 38 \\
\hline \multirow[t]{6}{*}{ AGREEABLENESS } & Trust & 73 & 74 & 68 & 74 \\
\hline & Morality & 64 & 67 & 64 & 61 \\
\hline & Altruism & 54 & 61 & 56 & 64 \\
\hline & Cooperation & 70 & 77 & 66 & 78 \\
\hline & Modesty & 63 & 52 & 55 & 61 \\
\hline & Sympathy & 72 & 73 & 68 & 70 \\
\hline \multirow[t]{6}{*}{ CONSCIENTIOUSNESS } & Self-Efficacy & 38 & 51 & 50 & 47 \\
\hline & Orderliness & 55 & 59 & 58 & 59 \\
\hline & Dutifulness & 52 & 67 & 61 & 59 \\
\hline & Achievement-Striving & 47 & 45 & 47 & 48 \\
\hline & Self-Discipline & 60 & 59 & 57 & 59 \\
\hline & Cautiousness & 48 & 63 & 55 & 63 \\
\hline \multicolumn{6}{|l|}{ OPENNESS TO } \\
\hline \multirow[t]{6}{*}{ EXPERIENCE } & Imagination & 16 & 24 & 26 & 29 \\
\hline & Artistic Interests & 39 & 47 & 43 & 53 \\
\hline & Emotionality & 56 & 53 & 56 & 63 \\
\hline & Adventurousness & 70 & 61 & 60 & 63 \\
\hline & Intellect & 45 & 57 & 54 & 59 \\
\hline & Liberalism & 65 & 72 & 69 & 70 \\
\hline
\end{tabular}

